J. Clin. Chem. Clin. Biochem.

Vol. 17, 1979, pp. 65-70

\title{
Neue enzymatische Lactatbestimmung: Methodische Aspekte und Probengewinnung ${ }^{1}$ )
}

Von W. Schwab

Städtische Krankenanstalten Karlsruhe, Medizinisch-diagnostisches Institut (Direktor Dr. H. Wüst), Karlsruhe,

W. Tritschler, A. Ch. Kessler und W. Bablok

Boehringer Mannheim GmbH, Diagnostica Forschung, Mannheim

(Eingegangen am 24. April/18. September 1978)

Zusammenfassung: Mit einem neuen enzymatischen Test kann der Lactatgehalt im enteiweißten Blut und im Plasma rasch und sicher bestimmt werden. Bei der „Plasmamethode“ (Verdünnungsgrenze $18 \mathrm{mmol} / \mathrm{l}$ ) war auch mit hohen Lactatkonzentrationen die Reaktion bei Raumtemperatur in 10 Minuten abgelaufen; die „Methode mit Enteiweißung“ (Verdünnungsgrenze $10 \mathrm{mmol} / \mathrm{l}$ ) erforderte 15 Minuten. Präzision und Wiederfindung waren gut. Störungen durch Lipämie, Hämolyse, Bilirubin, Metabolite, Enzyme und Pharmaka wurden nicht beobachtet. Kurze, venöse Stauung hatte keinen Einfluß auf den Lactatgehalt. Eine rasche Glykolysehemmung war durch die Kombination von Glykolysehemmer mit sofortiger Kühlung des abgenommenen Blutes im Eisbad möglich.

Die niedrigsten Plasma-Lactat-Werte gaben unter diesen Bedingungen Natriumfluorid/EDTA und danach Kaliumfluorid/EDTA. Zwischen 30 Minuten und 2 Stunden nach Blutabnahme war bei allen Glykolysehemmern die Lactatkonzentration konstant. Nach dem Abzentrifugieren der Erythrocyten konnte das Plasma - ohne daß sich die Lactatkonzentration änderte - bis zu 6 Tage bei Raumtemperatur aufbewahrt werden.

Mit der „Plasmamethode“ wurden zwischen 8 und 23\% höhere Werte als mit der „Methode mit Enteiweißung“ gefunden. Diese Unterschiede lassen sich auf einen variablen und relativ niedrigen Lactatgehalt in den Erythrocyten zurückführen.

New enzymatic determination of lactate: Methodological aspects and sampling technique

Summary: The lactate concentration in deproteinized blood and in plasma can be determined rapidly and reliably with a new enzymatic test. When the "plasma method" was used, the reaction ran to completion in 10 minutes at room temperature even at high lactate concentrations (up to $18 \mathrm{mmol} / \mathrm{l}$ ), whereas it required 15 minutes at room temperature when deproteinized blood was used (linear range up to $10 \mathrm{mmol} / \mathrm{l}$ ). Both precision and recovery were good. No when deproteinized blood was used (linear range up to $10 \mathrm{mmol} / \mathrm{l}$ ). Both precision and recovery were good. No interference by lipemia, hemolysis, bilirubin, metabolites, enzymes, or drugs was observed. Brief venous stasis was found to have no effect on the lactate level. Glycolysis can be speedily inhibited by the addition of an appropriate inhibitor to the specimen, which must be cooled in an ice-bath immediately after being obtained. Under these conditions, the lowest plasma lactate values were found with sodium fluoride/EDTA and the second-lowest with potassium fluoride/EDTA. The lactate concentration remained constant, irrespective of the glycolysis inhibitor used, between 30 minutes and 2 hours after blood sampling. Plasma can be stored up to 6 days at room temperature with no effect on the lactate concentration.

The "plassma method" gave values $8-23 \%$ higher than the method using deproteinized blood. These differences are due to a relatively low but variable lactate content of the erythrocytes.

i) Auszugsweise vorgetragen am Kongreß der Deutschen Gesellschaft für Laboratoriumsmedizin, Berlin, 15.-19. Mai 1977. 


\section{Einleitung}

Mit einer neuen enzymatischen Methode ist die Lactatbestimmung im enteiweißten Überstand von Blut und in Plasma möglich. Die vorliegende Studie befaßt sich mit methodischen Aspekten dieses Tests und mit dem Problem der Probengewinnung. Darunter fällt der Einfluß der venösen Stauung sowie die Frage nach der Glykolysehemmung und der Probenstabilität.

\author{
Material und Methoden \\ Testprinzip \\ $L-(+)-$ Lactat $+\mathrm{NAD}^{+} \frac{\text { Lactat- }}{\underset{\text { Dehydrogenase }}{\longrightarrow}}$ Pyruvat $+\mathrm{NADH}+\mathrm{H}^{+}$ \\ Pyruvat $+L$-Glutamat $\frac{\text { Glutamat-Pyruvat- }}{\text { Transaminase }} L$-Alanin $+2-$-Oxo- \\ glutarat
}

Die durch Lactat-Dehydrogenase (E.C. 1.1.1.27) katalysierte Oxidation von Lactat führt zu Pyruvat und NADH, dessen Absorption gemessen wird. Um das Gleichgewicht der Reaktion auf die rechte Seite zu verschieben, werden das entstehende Pyruvat sowie die Wasserstoff-Ionen durch eine Glutamat-Pyruvat-Transaminase (E. C. 2.6.1.2)-katalysierte Glutamat-PyruvatTransarninierung bzw. durch den stark alkalischen Natriumcarbonat-Puffer abgefangen.

\section{Reagenzien}

Monotest Lactat, Precinorm S, Precipath S, Kaliumfluorid/ $\mathrm{K}_{2}$-EDTA (Zusatzreagenz für die Lactatbestimmung), Hilfsreagenzien sowie eingesetzte Substrate und Enzyme entstammten dem Lieferprogramm von Boehringer Mannheim GmbH, D-6800 Mannheim 31, Validate A und Validate N dem der Fa. Gödecke, D-7800 Freiburg. Mit Natriumfluorid/ EDTA präparierte Probenröhrchen wurden von der Fa. Sarstedt, D-5223 Rommelsdorf, Monoiodacetat- $\mathrm{Na} / \mathrm{K}_{2}$ EDTA beschichtete Kunststoffröhrchen von der $\mathrm{Fa}$. Greiner, D-7440 Nürtingen, bezogen.

\section{Geräte}

Spektral-Photometer Zeiss PMQ II bzw. Photometer Eppendorf Modell $1101 \mathrm{M}$ mit Thermostat, Wechselautomatik und Registriereinrich tung; Messung bei $365 \mathrm{~nm}$, capilettor (Labora Mannheim GmbH, D-6800 Mannheim); Mikro-Häma tokritZentrifuge, Typ 912 (Heraeus-Christ, D-3360 Osterode).

Methoden

Lactatbestimmung im enteiweißten Blut (,Methode mit Enteiweißung")

$0,5 \mathrm{ml}$ Blut wird zu 0,5 ml Perchlorsäure (eiskalt, etwa $1,0 \mathrm{~mol} / \mathrm{l}$ ) zugegeben. Nach sofortigem Mischen wird die Suspension 10 Minuten bei $3000 \mathrm{U} / \mathrm{min}(3000 \mathrm{~g})$ zen trifugiert. Die Durchführung der Bestimmung erfolgt anschließend nach Vorschrift des Herstellers (Messung gegen Probenleerwert).

\section{Lactatbestimmung im Plasma (,Plasmamethode“)}

$2 \mathrm{ml}$ Blut werden mit festem Glykoly sehemmer/Antikoagulans bzw. 2 Tropfen flüssigem KF/EDTA-Reagenz gemischt und . 10 Minuten bei $3000 \mathrm{U} / \mathrm{min}$ zentrifugiert. Die Durchführung der Bestimmung erfolgt anschließend nach Vorschrift des Herstellers (Messung gegen Probenleerwert). Die Verdünnung des Blutes durch flüssiges Reagenz wird berücksichtigt.

\section{Lactatbestimmung in Erythrocyten}

Blut wird in gekühlte Probenröhrchen (Eisbad) mit Glykolysehemmer/EDTA gegeben, gemischt und nach 30 Minuten Stehen 5 Minuten bei $3000 \mathrm{U} / \mathrm{min}$ zentrifugiert. Das Plasma wird ab- pipettiert, und die Ery throcyten werden in einer Mikrozentrifuge bei $12000 \mathrm{U} / \mathrm{min}(8000 \mathrm{~g}) 4$ Minuten zentrifugiert. In ein Reagenzglas werden $400 \mu \mathrm{l}$ Perchlorsäure (eiskalt, etwa $0,6 \mathrm{~mol} / \mathrm{l}$ ) vorgelegt, $200 \mu \mathrm{l}$ Ery throcy ten-Konzentrat mit capilettor zugegeben, gemischt und die Suspension mit einer Mikrozentrifuge 2 Minuten zentrifugiert. Zur Lactatbestimmung werden $200 \mu \mathrm{l}$ des Überstandes eingesetzt.

Zur Berechnung des Verdünnungsfaktors wurde die Dichte des Erythrocyten-Konzentrats bestimmt (Mittel aus 5 Messungen: $1100 \mathrm{~g} / 1)$; der Wassergehalt wurde mit 70\% eingesetzt.

\section{Versuchsdurch führung}

\section{Einfluß der Stauung}

10 Probanden wurde, nach 30 Minuten körperlicher Ruhe im Sitzen, aus der ungestauten Armvene Blut entnommen. Im Anschluß daran wurde $30 \mathrm{~s}$ (5 Probanden) und $2 \mathrm{~min}$ (5 Probanden) mit einem Druck, der zwischen dem individuellen systolischen und diastolischen Blutdruck lag, gestaut. Nach Lösen der Stauung wurde $z$ wischen $2 \mathrm{~s}$ und $10 \mathrm{~min}$ zu festgelegten Zeitpunkten 9 mal Blut mit einer 2 ml-Spritze entnommen und sofort in gekühlte Probenröhrchen (Eisbad) mit KF/EDTA überführt. Nach der letżten Blutabnahme einer Serie wurden die Proben zentrifugiert und der Lactatgehalt in den Plasmen aus Dreifachbestimmungen ermittelt.

\section{Einfluß von Temperatur und Glykolysehemmer}

Ein Proband wurde durch 15 Minuten Dauerlauf körperlịch belastet. Das anschließend abgenommene Blut wurde in gekühlte (Eiswasser, etwa $5^{\circ} \mathrm{C}$ ) sowie ungekühlte $\left(22-24^{\circ} \mathrm{C}\right.$ ) Probengefäße mit 3 verschiedenen Glykolysehemmern verteilt. Gleichzeitig wurde eine Blutprobe mit Perchlorsäure enteiweißt.

Die Blutproben wurden bei $5^{\circ} \mathrm{C}$ und bei Raumtemperatur gelagert und in den einzelnen Plasmen der Lactatgehalt zwischen 12 Minuten und 48 Stunden nach Blutabnahme aus Doppelbestimmungen ermittelt. Um die Reproduzierbarkeit der Daten zu prüfen, wurde der Versuch nach 4 Wochen am selben Probanden wiederholt. Die niedrigsten gefundenen Plasma-Lactatkonzentrationen $(9,0$ bzw. 11,5 mmol/1 in NaF/EDTA bei etwa $5{ }^{\circ} \mathrm{C}, 30$ Minuten nach Blutabnahme gemessen) wurden als Basiswert genommen und für alle anderen Lactatkonzentrationen die Differenzen $\Delta c=c_{\text {gemessen }}-c_{\text {Basiswert }}$ berechnet (Abb. 3).

Vergleich der Methode mit Enteiweißung mit der Plasmamethode

81 Blutproben wurden enteiweißt bzw. gleichzeitig in gekühlte (etwa $5^{\circ} \mathrm{C}$ ) mit Kaliumfluorid/EDTA versehene Probengefäße gegeben, nach etwa 30 min zentrifugiert und der Lactatgehalt aus Doppelbestimmungen ermittelt.

\section{Ergebnisse und Diskussion}

\section{Analy tische Eigenschaften}

\section{Meßbereich und Wiederfindung}

Für die Plasmamethode zeigten wäßrige Lactatlösungen bis zu einer Konzentration von $18 \mathrm{mmol} / 1$ eine lineare Konzentrationsbeziehung. Bei der Methode mit Enteiweißung ergaben mit Perchlorsäure $(0,6 \mathrm{~mol} / \mathrm{l})$ verdünnte Lactatlösungen einen linearen Meßbereich bis $10 \mathrm{mmol} / \mathrm{l}$ (Abb. 1). Mit Lactatlösung aufgestockte Plasmen bzw. enteiweißte Úberstände von Blut gaben gleiche Resultate. Der für die Methode mit Enteiweißung gefundene kleinere lineare Meßbereich ist $z$. $T$. auf die enzyminhibierende Wirkung der Perchlorat-Ionen zu= rückzuführen. 'Durch die Neutralisation des enteiweißten Uberstandes wird außerdem die aktivierende Wirkung der Carbonat-Ionen auf die Glutamat-Pyruvat-Transaminase vermindert. Im linearen Konzentrationsbereich 


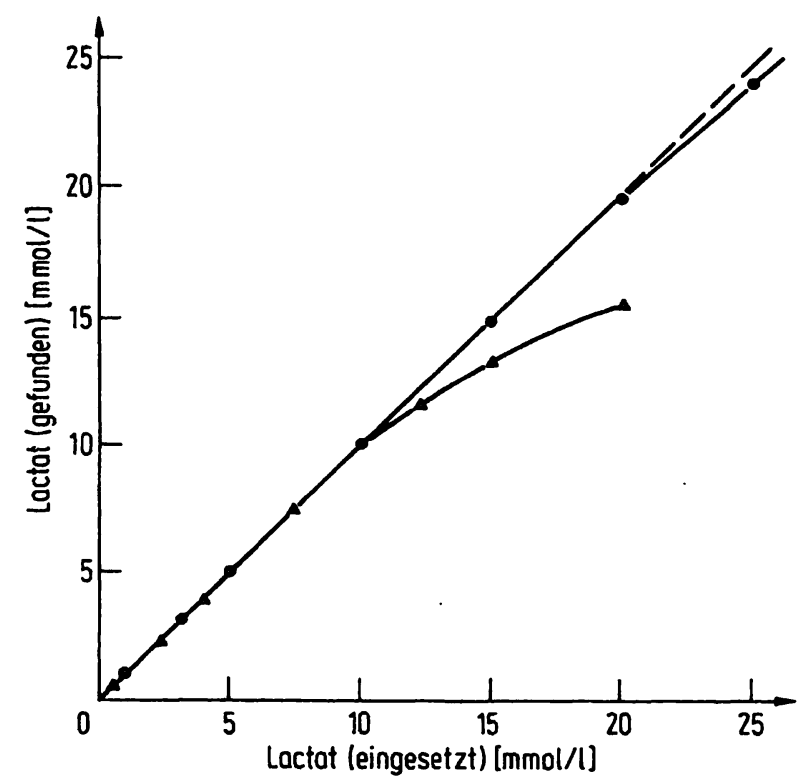

Abb. 1. Meßbereich und Wiederfindung in Standardlösungen für Plasmamethode und Methode mit Enteiweißung. - — Plasmamethode, $\triangle$ _ Methode mit Enteiweißung, - - Bezugsgerade.

lagen die Wiederfindungsraten für beide Methoden zwischen 97 und 100\%.

In den Kontrollseren Precinorm S, Precipath S, Validate $A$ und Validate $\mathrm{N}$ wurden die Sollwerte zu 97 bis $101 \%$ wiedergefunden.

\section{Reaktionsverlauf}

Abbildung 2 zeigt die Absorptions-Zeitdiagramme der Plasmamethode und der Methode mit Enteiweißung. Für die Plasmamethode ergeben sich dabei um etwa die Hälfte kürzere Inkubationszeiten als für die Methode mit Enteiweißung. Bei hohen Lactatkonzentrationen im Plasma (bis $18 \mathrm{mmol} / \mathrm{l}$ ) waren bei $20-25^{\circ} \mathrm{C} 10 \mathrm{Minu}$ ten, bei $30^{\circ} \mathrm{C} 6$ Minuten Inkubationszeit erforderlich. Für beide Methoden ist eine Messsung gegen den Probenleerwert notwendig, da es durch die Instabilität des NAD im stark alkalischen Medium des Testansatzes zu konstanten Absorptionszunahmen (etwa 0,001 $\Delta \mathrm{A} / \mathrm{min}$ ) kommt.

Bei $37^{\circ} \mathrm{C}$ sollte nicht inkubiert werden, da bei dieser Temperatur das gleichsinnige Verhalten von Probe und Probenleerwert nicht mehr gewährleistet ist.

\section{Präzision}

Für Plasmamethode und Methode mit Enteiweißung wurden Fünffach-Analysen je einer Probe aus 3 Konzentrationsbereichen an 5 Tagen durchgeführt (Tab. 1).

Beide Methoden zeigten eine gute Präzision in der Serie und von Tag zu Tag.

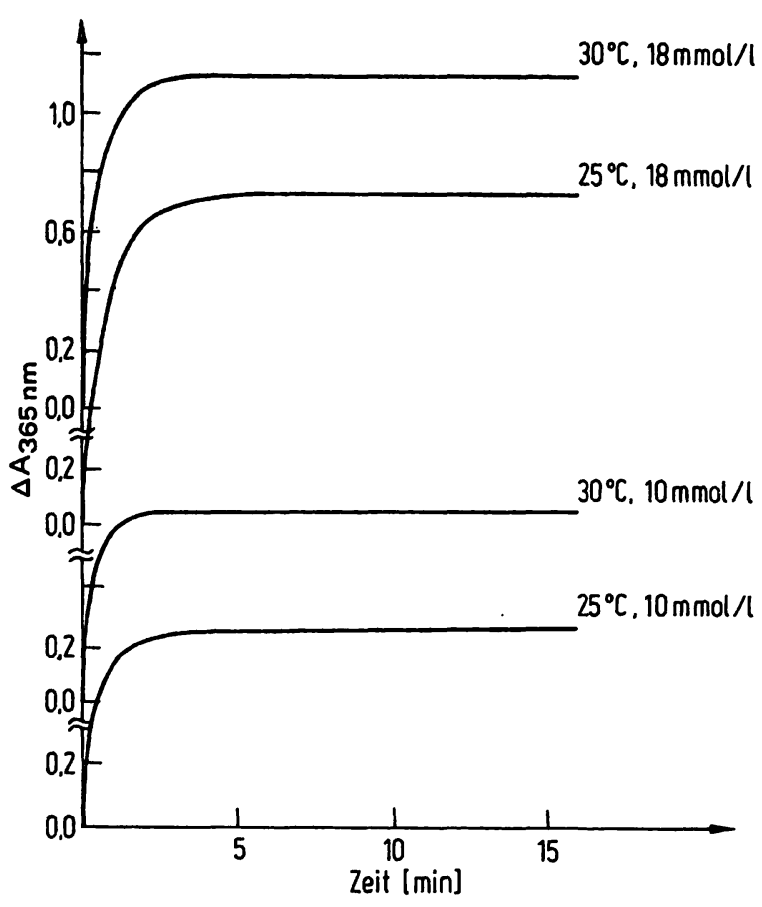

Abb. 2a

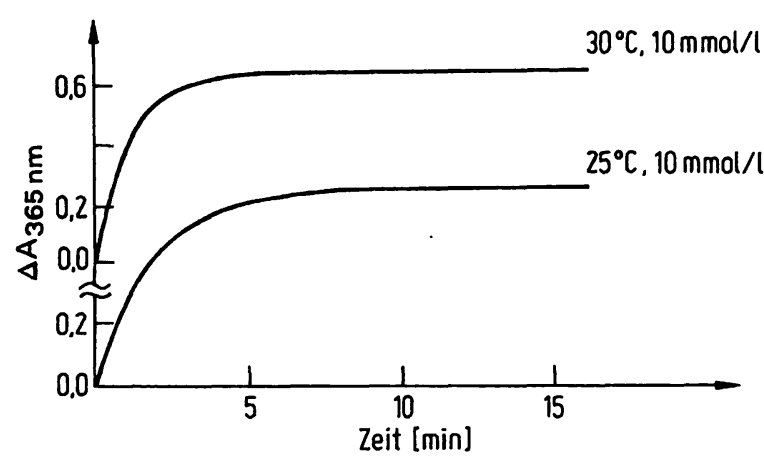

Abb. 2b

Abb. 2. a) Umsatzkurven für die Plasmamethode bei $25^{\circ} \mathrm{C}$ und $30^{\circ} \mathrm{C}$

b) Umsatzkurven für die Methode mit Enteiweißung.bei $25^{\circ} \mathrm{C}$ und $30^{\circ} \mathrm{C}$.

Tab. 1. Präzision der Methoden.

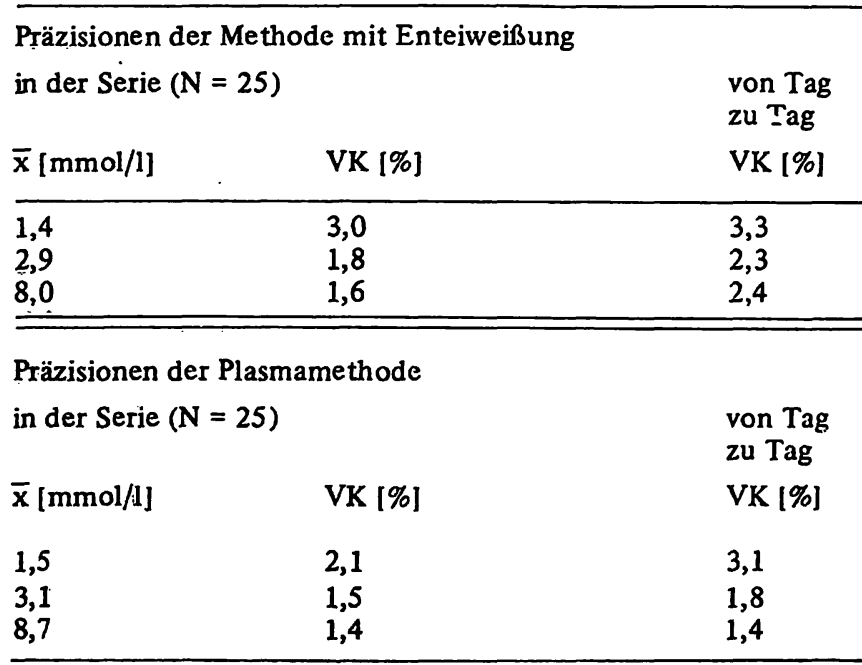




\section{Störungen}

Lipämie: Triglyceridkonzentrationen von $11 \mathrm{mmol} / 1$ Plasma waren ohne Einfluß auf das Ergebnis.

Hämolyse: Hämoglobinkonzentrationen im Plasma bis $125 \mu \mathrm{mol} / 1 \mathrm{Hb} / 4$ störten nicht.

Bilirubin: Bilirubinkonzentrationen bis $340 \mu \mathrm{mol} / 1$ Plasma beeinflußten die Bestimmung nicht.

Metabolite und Enzyme: Verschiedene Substrate, Enzyme und Enzymkombinationen störten die Bestimmung nicht (Tab. 2).

Pharmaka: Die auf ein Volumen von 51 bezogene therapeutische Tages-Maximaldosis wurde in Serum gelöst. Die 34 geprüften Wirkstoffe störten den Test nicht (Tab. 3).

\section{Einfluß der Stauung}

Uber den Einfluß der venösen Stauung auf die Lactatkonzentration sind in der Literatur widersprüchliche Angaben gemacht worden $(1,2)$.

Unsere Untersuchungen an 10 Probanden zeigten, daß eine Stauung von 30 Sekunden keinen Einfluß auf den Lactatgehalt hat:

Die Abweichungen vom Ausgangswert (Blutabnahme ungestaut, etwa $1 \mathrm{mmol} / 1$ Lactat) betrugen weniger als $0,04 \mathrm{mmol} / 1$ Lactat und lagen damit im Bereich der Meßungenauigkeit der Methode.

Nach 2 Minuten Stauung war eine leichte Zunahme der Lactatkonzentration bis etwa $0,1 \mathrm{mmol} / \mathrm{l} \mathrm{zu}$ beobachten. Die Abweichungen zum Ausgangswert unterlagen jedoch starken Schwankungen. Sicherheitshalber

Tab. 2. Metabolite und Enzyme, deren Anwesenheit im Bestimmungsansatz keine nachweisbare Störung zeigte.

\begin{tabular}{|c|c|c|}
\hline \multirow[t]{2}{*}{ Metabolite bzw. Enzyme } & \multicolumn{2}{|c|}{$\begin{array}{l}\text { max. eingesetzte } \\
\text { Konzentrationen bzw. } \\
\text { Aktivitäten }\end{array}$} \\
\hline & {$[\mathrm{mmol} / \mathrm{l}]$} & {$[\mathrm{kU} / \mathrm{l}]$} \\
\hline $\begin{array}{l}\text { Pyruvat } \\
D, L-3 \text {-Hydroxybutyrat } \\
D-(-) \text {-Lactat }\end{array}$ & $\begin{array}{r}2 \\
10 \\
10\end{array}$ & \\
\hline $\begin{array}{l}\text { Lactat-Dehydrogenase } \\
\text { (Muskel, Herz) }\end{array}$ & & 2,5 \\
\hline Glutamat-Pyruvat-Transaminase & & 50 \\
\hline Glutamat-Dehydrogenase & & 2 \\
\hline $\begin{array}{l}\text { Lactat-Dehydrogenase/ } \\
\text { Glutamat-Pyruvat-Transaminase }\end{array}$ & & $2 ; 7$ \\
\hline $\begin{array}{l}\text { Lactat-Dehydrogenase/ } \\
\text { Glutamat-Dehydrogenase }\end{array}$ & & 2,$5 ; 0,5$ \\
\hline $\begin{array}{l}\text { Glutamat-Pyruvat-Transaminase/ } \\
\text { Glutamat-Dehydrogenase }\end{array}$ & & $7 ; 0 ; 5$ \\
\hline $\begin{array}{l}\text { Lactat-Dehydrogenase/ } \\
\text { Glutamat-Dehydrogenase/ } \\
\text { Glutamat-Pyruvat-Transaminase }\end{array}$ & & $2 ; 0,4 ; 6$ \\
\hline
\end{tabular}

Tab. 3. Wirkstoffe, deren Anwesenheit im Bestimmungsansatz keine nachweisbare Störung zeigte.

\begin{tabular}{lc}
\hline Wirkstoffe & $\begin{array}{c}\text { eingesetzte Wirkstoff- } \\
\text { konzentrationen } \\
\text { mg/l Serum bzw. } \\
\text { ml/1 Serum* }\end{array}$ \\
& 1400 \\
& 600 \\
\hline Acetylsalicylsäure & $4^{*}$ \\
Acidum ascorbicum & $4^{*}$ \\
Adipiodon-Meglumin $(0,5 \mathrm{~g} / \mathrm{ml})$ & 1200 \\
Amidotrizoat-Meglumin $(0,65 \mathrm{~g} / \mathrm{ml})$ & 4000 \\
Ampicillin & 60 \\
Bisacodyl & 180 \\
Buformin & 400 \\
Carbochromen & 6 \\
Chloramphenicol & 300 \\
Chlordiazepoxid & $100^{*}$ \\
Coffein & 40 \\
Dextran (6\%) & 400 \\
Dipyridamol & 100 \\
Ethaverin & $200^{*}$ \\
Furosemid & 40 \\
Gelatine (30 g/l) & 80 \\
Indometacin & 400 \\
Metaqualon & 60 \\
Methyldopum & 800 \\
Nitrofurantoin & 30 \\
Noramidopyrin-methansulfonat & 120 \\
Oxazepam & 600 \\
Oxiphenbutazon & 600 \\
Oxytetracyclin & 120 \\
Paracetamol & 60 \\
Phenazopyridin & 2 \\
Phenobarbital & 160 \\
Phenprocoumon & 200 \\
Phenytoin & 40 \\
Probenecid & 120 \\
Procain & 150 \\
Pyrithioxin & 320 \\
Rifampicin & \\
Sulfamethoxazol & \\
& \\
& \\
&
\end{tabular}

sollte nicht viel länger als 30 Sekunden venös gestaut werden, da nach längerer Stauung ein Einfluß im Einzelfall möglich ist.

\section{Einfluß von Temperatur und Glykolysehemmer}

Der Einfluß der Temperatur auf die in vitro-Lactatproduktion der Blutkörperchen ist bekannt (3). Für die Lactatbestimmung wird deshalb die sofortige Enteiweißung von Blut oder die Probennahme in natriumfluorid-hal tige Probenröhrchen und Eiskühlung der Proben bis zur sofortigen Untersuchung empfohlen (4). Unsere Untersuchungen zum Einfluß von Temperatur und Glykolysehemmer auf den Lactatgehält sind in Abbildung 3 dargestellt.

Es zeigte sich, daß bei Lagerung der Blutproben zwischen 30 Minuten und 2 Stunden nach Blutabnahme keine wesentlichen Änderungen der Lactatkonżentrationen auftreten.

Die Glykolysehemmung mit Fluoriden erfolgt bei $5^{\circ} \mathrm{C}$ schneller als bei $22^{\circ} \mathrm{C}$. Monoiodacetat hemmt die Gly. kolyse temperaturunabhängig. Die unterschiedlichen Werte bei Einsatz von Kạlium- und Natriumfluorid las- 


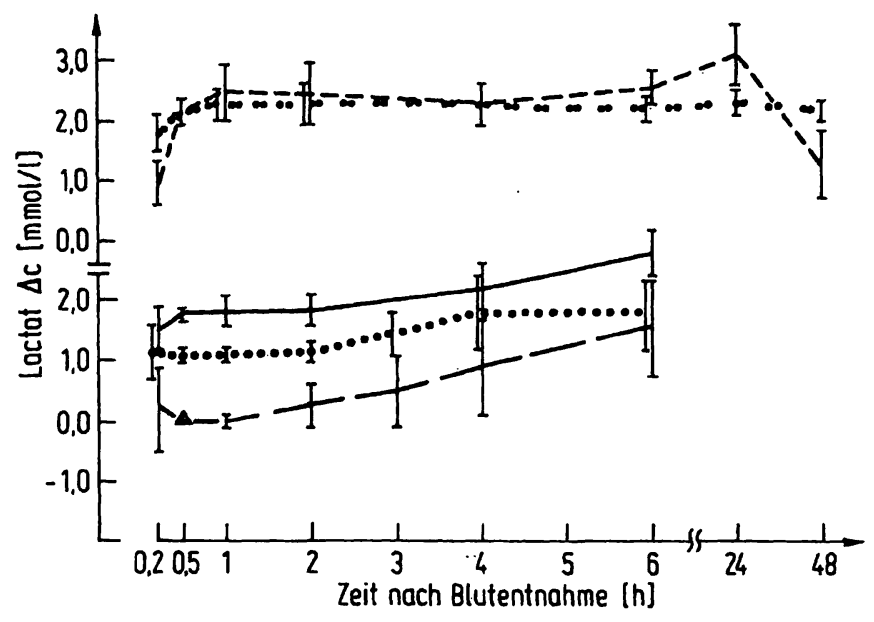

Abb. 3. Einfluß von Temperatur und Glykolysehemmer auf den Lactatgehalt von Blut.

A Basiswert $(=$ mittlere Lactatkonzentration von etwa $10 \mathrm{mmol} / \mathrm{l}$ nach 30 Minuten mit NaF/EDTA bei etwa $5^{\circ} \mathrm{C}$ ).

$\Delta \dot{c}$ Konzentrationsabweichungen vom Basiswert:

- in NaF/EDTA bei etwa $5^{\circ} \mathrm{C}$ bzw. - - - bei etwa $22^{\circ} \mathrm{C}, \bullet \cdots \cdots \bullet$ in KF/EDTA bei etwa $5^{\circ} \mathrm{C}$ bzw.

$\bullet \bullet \bullet$ bei etwa $22^{\circ} \mathrm{C}, \longrightarrow$ in Monoiodacetat/EDTA bei etwa $5^{\circ} \mathrm{C}$ und etwa $22^{\circ} \mathrm{C}$.

I Werte aus Erst- und Zweituntersuchung.

sen sich z. T. durch die unterschiedlich starke Schrumpfung der Erythrocyten erklären, wodurch das Plasma verschieden stark verdünnt wird. Folgende Daten stützen diese Vermutung: Der Hämatokrit von 6 Blutproben mit Kaliumfluorid-EDTA-Gemisch lag um 13-19\% niedriger, der von Blutproben mit NatriumfluoridEDTA-Gemisch um $21-31 \%$ niedriger als der von EDTABlut. Die Lagerungszeit der Blutproben (bis 2 Stunden) sowie deren Temperatur $\left(5^{\circ} \mathrm{C}\right.$ und $\left.25^{\circ} \mathrm{C}\right)$ hatten keinen Einfluß auf den Hämatokrit. Demnach schrumpfen die Ery throcy ten in natriumfluorid-hal tigem Blut stärker als in Blut mit Kaliumfluorid.

Dadurch steigt der Wassergehalt des Plasmas und man erhält mit Natriumfluorid/EDTA-Gemișch dementsprechend niedrigere Lactatwerte.

Außerdem ist wahrscheinlich ein unterschiedliches Diffusionsverhalten der beiden Fluoride für die unterschiedliche Glykolysehemmung verantwortlich.

Die absolut niedrigsten Lactatkonzentrationen wurden nach Enteiweißung von Blut mit eiskalter Perchlorsäure erhalten. Die Lactatwerte lagen um etwa $8 \%$ unter den mit Natriumfluorid und Kühlung der Proben erhaltenen Plasmà-Konzentrationen (Näheres siehe unțen).

Bei Raumtemperatur gelagerte Plasmen mit Kaliumfluorid/EDTA zeigten während 6 Tagen keine Änderung der Lactatkonzentration.

Vergleich der Plasmamethode mit der Methode mit Enteiweißung

Bei Vergleichsuntersuchungen an 81 Blutproben gab die Plasmamethode unter definierten Bedingungen (Proben-

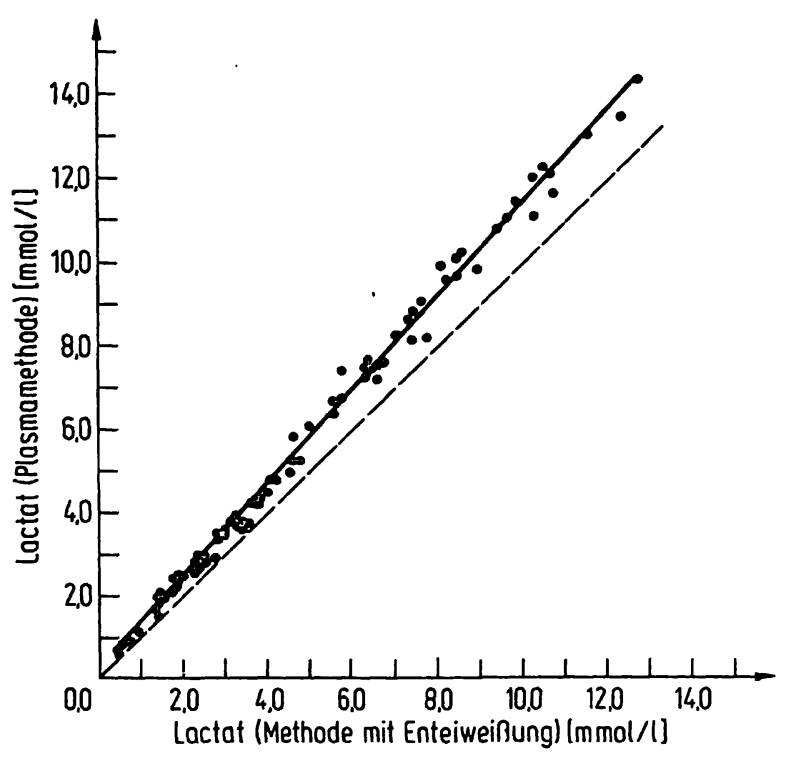

Abb. 4. Vergleich der Plasmamethode mit der Methode mit Enteiweißung für 81 Blutproben.

$y=0,15+1,13 x,-$ Bezugsgerade $y=x$.

kühlung, Kaliumfluorid/EDTA) ${ }^{2}$ ) höhere Werte als die Methode mit Enteiweißung (Abb. 4). Die Differenzen lagen für das 5. Perzentil bei $8 \%$ und für das 95 . Perzentil bei 23\%; der Median betrug 13\%. Da bei der Methode mit Enteiweißung der mittlere Wassergehalt des Blutes (80\%) sowie dessen mittlere Dichte $(D=1060 \mathrm{~g} / \mathrm{l})$ bei der Berechnung der Lactatkonzentration berücksichtigt sind, muß der Unterschied zur Plasmamethode mit einem niedrigen Lactatgehalt in den Erythrocyten erklärt werden: Bei der Enteiweißung von Blut werden die Erythrocyten zerstört und der niedrigere Lactatgehalt des Erythrocyten-Wassers verdünnt die Lactatkonzentration im Plasma-Wasser.

Wir bestimmten daher den Lactatgehalt in Plasma, Erythrocyten und in enteiweißtem Blut aus Proben von 12 Probanden und versuchten rechnerisch $z u$ beweisen, daß die Unterschiede zwischen Plasmamethode und Methode mit Enteiweißung auf einen relativ niedrigen Lactatgehalt der Ery throcy ten zurückzuführen sind (Tab. 4).

Mit Hilfe des Hämatokrits wurden die gemessenen Lactatkonzentrationen in Plasma und Erythrocyten entsprechend ihren Volumenanteilen auf Blut umgerechnet. Die Summe der für Plasma und Erythrocyten berechneten Lactatkonzentrationen stimmte gut mit der gemessenen Lactatkønzentration nach Enteiweißung von Blut überein.

Der relative Lactatgehalt der Erythrocyten lag zwischen 0,10 und 0,38 , der von Plasma entsprechend zwischen

2) Obwohl mit Natriumfluorid als Glykolysehemmer und Kühlung des abgenommenen Blutes die niedrigsten PlasmaLactatwerte erhalten wurden (Abb. 3), gaben wir Kaliumfluorid den Vorzug, weil hierbei die Schrumpfung der Erythrocyten und die Hämolyse geringer sind. 
Tab. 4. Bestimmung des relativen Lactatgehaltes in Erythrocyten.

\begin{tabular}{|c|c|c|c|c|c|c|c|c|}
\hline \multirow{2}{*}{$\begin{array}{l}\text { Glykolyse- } \\
\text { hemmer/ } \\
\text { Antikoagulans }\end{array}$} & \multicolumn{3}{|c|}{ Lactatkonzentration gemessen [mmol/l] } & \multirow[t]{2}{*}{ Hämatokrit } & \multicolumn{3}{|c|}{ Lactatgehalt berechnet [mmol/1] } & \multirow{2}{*}{$\begin{array}{l}\text { Relativer } \\
\text { Lactat- } \\
\text { gehalt der } \\
\text { Erythro- } \\
\text { cyten }\end{array}$} \\
\hline & Plasma & $\begin{array}{l}\text { Erythro- } \\
\text { cyten }\end{array}$ & $\begin{array}{l}\text { Blut mit } \\
\text { Enteiweißung }\end{array}$ & & $\begin{array}{l}\text { Anteil in } \\
\text { Plasma (P) }\end{array}$ & $\begin{array}{l}\text { Anteil in } \\
\text { Erythro- } \\
\text { cyten (E) }\end{array}$ & $P+E$ & \\
\hline $\mathrm{NaF} / \mathrm{EDTA}$ & $\begin{array}{l}0,76 \\
1,98 \\
3,65 \\
4,33 \\
5,77 \\
7,51^{*}\end{array}$ & $\begin{array}{l}0,21 \\
0,67 \\
2,78 \\
2,53 \\
3,93 \\
5,04\end{array}$ & $\begin{array}{l}0,60 \\
1,40 \\
3,25 \\
3,79 \\
4,72 \\
6,54\end{array}$ & $\begin{array}{l}0,29 \\
0,37 \\
0,24 \\
0,30 \\
0,36 \\
0,31\end{array}$ & $\begin{array}{l}0,54 \\
1,25 \\
2,76 \\
3,04 \\
3,70 \\
5,18\end{array}$ & $\begin{array}{l}0,06 \\
0,25 \\
0,67 \\
0,76 \\
1,42 \\
1,56\end{array}$ & $\begin{array}{l}0,60 \\
1,50 \\
3,43 \\
3,80 \\
5,12 \\
6,74\end{array}$ & $\begin{array}{l}0,10 \\
0,18 \\
0,21 \\
0,20 \\
0,30 \\
0,24\end{array}$ \\
\hline $\mathrm{KF} / \mathrm{EDTA}$ & $\begin{array}{l}0,67 \\
1,14 \\
2,21 \\
2,55 \\
2,82 \\
6,86\end{array}$ & $\begin{array}{l}0,23 \\
0,40 \\
1,09 \\
1,65 \\
1,89 \\
4,80\end{array}$ & $\begin{array}{l}0,47 \\
0,83 \\
1,81 \\
2,24 \\
2,50 \\
6,10\end{array}$ & $\begin{array}{l}0,44 \\
0,39 \\
0,41 \\
0,29 \\
0,38 \\
0,48\end{array}$ & $\begin{array}{l}0,38 \\
0,70 \\
1,30 \\
1,81 \\
1,75 \\
3,56\end{array}$ & $\begin{array}{l}0,10 \\
0,16 \\
0,45 \\
0,48 \\
0,72 \\
2,30\end{array}$ & $\begin{array}{l}0,48 \\
0,86 \\
1,75 \\
2,29 \\
2,47 \\
5,86\end{array}$ & $\begin{array}{l}0,21 \\
0,19 \\
0,25 \\
0,21 \\
0,29 \\
0,38\end{array}$ \\
\hline
\end{tabular}

* Berechnungsbeispiel: Anteil in Plasma: $\quad 7,51 \cdot(1,00-0,31) \mathrm{mmol} / \mathrm{l}=5,18 \mathrm{mmol} / \mathrm{l}$ Anteil in Erythrocyten: $\quad 5,04 \cdot 0,31 \mathrm{mmol} / 1=1,56 \mathrm{mmol} / 1$ Summe: $\quad(5,18+1,56) \mathrm{mmol} / 1=6,74 \mathrm{mmol} / 1$ (experimentell: $6,54 \mathrm{mmol} / \mathrm{l}$ )

Relativer Lactatgehalt in Ery throcy ten: $\frac{1,56 \mathrm{mmol} / 1}{6,54 \mathrm{mmol} / 1}=0,24$

0,90 und 0,62. Niedrige relative Lactatwerte in den Erythrocyten wurden bei Probanden ohne körperliche Belastung gefunden, während nach körperlicher Arbeit höhere Werte auftraten.

Die oben gefundenen Unterschiede zwischen der Methode mit Enteiweißung von Blut und der Plasmamethode lassen sich daher mit einem relativ niedrigen und variablen Lactatgehalt der Erythrocyten erklären.
Als Folgerung ergibt sich, daß weder die Methode mit Enteiweißung von Blut noch die Plasmamethode „richtige“ Lactatwerte gibt, da bei beiden der Lactatgehalt der Erythrocyten einen Einfluß auf das Ergebnis hat. Die Plasmamethode bietet gegenüber der Methode mit Enteiweißung jedoch Vorteile bei der Probengewinnung und bei der Testdurchführung, wo der Zeitbedarf pro Bestimmung erheblich niedriger ist. Dies kann in Notfallsituationen von Bedeutung sein.

\section{Literatur}

1. Braybrooke, J., Lloyd, B., Nattras, M. \& Alberti, K. G. M. M. (1975), Ann. Clin. Biochem. 12, 252-254.

2. Noll, F. (1974), in Methoden der enzymatischen Analyse (Bergmeyer, H. U. ed.), 3. Aufl., S. 1521-1525. Verlag Chemie, Weinheim.

3. Racine, Ph., Klenk, H.-O. \& Kochsiek, K. (1975), diese Z. $13,533-539$.

4. Alderman, J. A. \& Cross, R. E. (1977), Clin. Chem. 23, 1917-1920.

Dr. rer. nat. W. Tritschler Boehringer Mannheim GmbH Diagnostica Forschung Sandhofer Straße 116 D-6800 Mannheim 31 